\title{
Avaliação do risco nutricional em crianças hospitalizadas: uma comparação da avaliação subjetiva global pediátrica e triagem nutricional STRONGkids com os indicadores antropométricos
}

\author{
Nutritional risk assessment in hospitalized children: a comparison \\ of pediatric subjective global assessment and STRONGkids screening tool \\ with anthropometric indicators
}

\author{
Luciana da Silveira Klein Campos ${ }^{1}$, Laura Dresch Neumann², Estela Iraci Rabito ${ }^{3}$, \\ Elza Daniel de Mello ${ }^{4}$, Juliana Paludo Vallandro ${ }^{4} \bowtie$ \\ Programa de Residência Multiprofissional Integrada em Saúde da Universidade Federal de Ciências da Saúde de Porto Alegre (UFCSPA). Porto Alegre, RS. \\ 2 Curso de graduação em Nutrição da UFCSPA. Porto Alegre, RS. \\ Departamento de Nutrição da Universidade Federal do Paraná (UFPR). Curitiba, PR. \\ ${ }^{4}$ Programa de Pós Graduação em Saúde da Criança e do Adolescente da Universidade Federal do Rio Grande do Sul (UFRGS). Porto Alegre, RS
}

\section{RESUMO}

Objetivos: Comparar a Avaliação Nutricional Subjetiva Global (ANSG) e a Triagem de Risco para Estado Nutricional e Crescimento (STRONGkids) com a avaliação antropométrica, na admissão hospitalar, e associá-las ao tempo de internação em crianças hospitalizadas.

Métodos: Estudo transversal com pacientes de 4 a 8,9 anos internados em um hospital pediátrico de Porto Alegre, Rio Grande do Sul. A amostragem foi realizada por conveniência, e a coleta de dados ocorreu entre junho e outubro de 2014. Não foram incluídos pacientes internados em Unidade de Terapia Intensiva e aqueles sem condições de alimentação por via oral. Foram coletadas informações gerais e socioeconômicas, e aplicados os questionários de ANSG e STRONGkids na admissão hospitalar. Dados clínicos e antropométricos foram coletados do prontuário eletrônico.

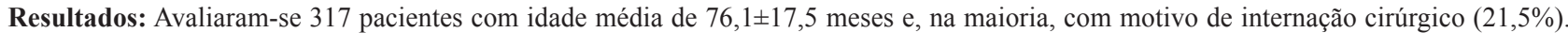
Segundo a avaliação antropométrica, $5 \%$ dos pacientes eram desnutridos, $74,1 \%$ eram eutróficos e 20,8\% apresentavam excesso de peso. Houve associação significativa entre a desnutrição classificada pela avaliação antropométrica e a desnutrição moderada e grave definida pela ANSG $(\mathrm{p}<0,001)$. Também houve associação significativa entre excesso de peso, eutrofia e desnutrição, classificados pela avaliação antropométrica, e risco nutricional baixo, médio e alto, respectivamente $(\mathrm{p}<0,001)$. Houve concordância significativa, porém muito fraca, entre a STRONGkids e a avaliação antropométrica (kappa $=0,148 ; \mathrm{p}=0,001)$.

Conclusões: Todas as avaliações feitas por meio das ferramentas testadas associaram-se (embora em fraca intensidade) com o tempo de internação hospitalar. Além disso, a STRONGkids apresentou maior concordância, embora ainda fraca, com a avaliação antropométrica quando comparada à ANSG. São necessários mais estudos verificando a concordância dessas ferramentas com outros métodos objetivos de avaliação nutricional.

DESCRITORES: avaliação nutricional; criança hospitalizada; desnutrição.

\section{ABSTRACT}

Aims: To compare the Subjective Global Nutritional Assessment (SGNA) and the Screening Tool for Risk on Nutritional Status and Growth (STRONGkids) protocols with anthropometric measurements at admission, and associate them to length of stay in hospitalized children.

Methods: Cross-sectional study with patients from four to 8.9 years admitted to a pediatric hospital in Porto Alegre, Rio Grande do Sul, Brazil. The sample was selected by convenience, and data collection occurred between June and October 2014. Patients in the Intensive Care Unit and those unable to feed orally were not included. We collected general and socioeconomic information and applied STRONGkids and SGNA protocols at admission. Clinical and anthropometric data were retrieved from electronic medical records.

Results: We evaluated 317 patients with a mean age of $76.1 \pm 17.5$ months, most of them admitted for surgery (21.5\%). According to anthropometric measurements, $5 \%$ of patients were malnourished, $74.1 \%$ had normal weight and $20.8 \%$ were overweight. There was a statistically significant association between malnutrition classified by anthropometric measurements and moderate and severe malnutrition defined by SGNA $(\mathrm{p}<0.001)$. There was also a significant association between overweight, normal weight and malnutrition classified by anthropometric measurements and low, medium and high nutritional risk, respectively $(\mathrm{p}<0.001)$. There was a statistically significant agreement, although very weak, between STRONGkids and anthropometric measurements (kappa $=0.148 ; \mathrm{p}=0.001$ ).

Conclusions: All protocols were associated (though in low intensity) to length of hospital stay. In addition, STRONGkids showed greater agreement, although still weak, with anthropometric measurements when compared to the SGNA. Further studies are needed to verify the agreement of these protocols with other objective methods of nutritional assessment.

KEY WORDS: nutrition assessment; child, hospitalized; malnutrition.

Recebido: setembro, 2015

Aceito: dezembro, 2015

Este artigo está licenciado sob forma de uma licença Creative Common 
Abreviaturas: ANSG, Avaliação Nutricional Subjetiva Global; STRONGkids, Triagem de Risco para Estado Nutricional e Crescimento; IMC/I, índice de massa corporal para idade; P/I, peso para idade; E/I, estatura para idade; OMS, Organização Mundial da Saúde.

\section{INTRODUÇÃO}

A desnutrição é um estado no qual uma deficiência ou desequilíbrio de energia, proteínas e/ou outros nutrientes provoca efeitos adversos mensuráveis na composição corporal e tecidual [1] e nos desfechos clínicos e funcionais [2]. Em crianças hospitalizadas, é considerada um fator de risco para desfechos desfavoráveis, tempo de internação prolongado, atraso de recuperação e maiores custos relacionados aos cuidados em saúde [3].

A fim de prevenir a desnutrição, especialmente a adquirida durante a internação hospitalar, o risco nutricional deve ser identificado precocemente, de preferência no momento da admissão, de modo que uma intervenção nutricional adequada possa ser iniciada [4]. Contudo, atualmente não há um consenso de qual o melhor método de avaliação do risco nutricional neste contexto [2]. Os diversos métodos de avaliação nutricional existentes não fornecem informações suficientes para a classificação do estado nutricional quando utilizados de forma isolada [5]. Desse modo, faz-se necessária a existência de um método abrangente que associe diferentes variáveis, identificando de maneira precisa os pacientes desnutridos [6].

Cinco ferramentas foram desenvolvidas recentemente para identificar o risco de deterioração da condição nutricional em crianças hospitalizadas [7-11]. Dentre elas, a Triagem de Risco para Estado Nutricional e Crescimento (Screening Tool for Risk on Nutritional Status and Growth - STRONGkids) consiste em um instrumento de triagem nutricional de simples utilização que aborda quatro elementos subjetivos: exame físico, identificação de doenças de risco nutricional, avaliação da ingestão alimentar e das alterações gastrointestinais e avaliação da perda de peso [10]. Já a Avaliação Nutricional Subjetiva Global (ANSG) é uma ferramenta mais complexa e que exige maior tempo de aplicação, abordando exame físico, dados antropométricos usuais e atuais da criança, avaliação da ingestão alimentar e das alterações gastrointestinais e identificação de doenças de risco nutricional. Essa ferramenta possui o objetivo de avaliar subjetivamente o estado nutricional dos pacientes, classificando as crianças em três diferentes categorias: bem nutridas, moderadamente desnutridas e gravemente desnutridas [8].
Considerando-se que ainda não existe um consenso sobre os métodos de avaliação do risco nutricional pediátrico, é necessária a identificação da ferramenta de maior acurácia para definir o risco de deterioração da condição nutricional, a fim de ajustá-la ao contexto clínico da criança hospitalizada. O presente estudo tem como objetivo comparar a ANSG específica para pediatria e a STRONGkids com a avaliação antropométrica, na admissão hospitalar, e associar esses diferentes métodos ao tempo de internação em crianças hospitalizadas.

\section{MÉTODOS}

Foi realizado um estudo transversal que incluiu crianças de 4,0 a 8,9 anos de idade internadas em um hospital pediátrico de Porto Alegre, capital do Rio Grande do Sul. A coleta de dados ocorreu entre junho e outubro de 2014. A amostragem foi realizada por conveniência, não tendo sido incluídos pacientes internados em Unidade de Terapia Intensiva e/ou que não se alimentavam por via oral. Foram excluídos os pacientes cujos pais e/ou responsáveis não concordaram com a participação na pesquisa. Aqueles que aceitaram participar assinaram um Termo de Consentimento Livre e Esclarecido. O projeto foi aprovado pelo Comitê de Ética em Pesquisa da Irmandade da Santa Casa de Misericórdia de Porto Alegre (parecer no 029.407 ), atendendo às diretrizes e normas reguladoras de pesquisas envolvendo seres humanos (Resolução 466/2012).

Os pacientes foram entrevistados nas unidades de internação em até 72 horas após a admissão hospitalar. Inicialmente, foram coletadas informações como sexo, idade e cor/raça do paciente, escolaridade do respondente e condições socioeconômicas da família, definidas através do Critério de Classificação Econômica Brasil [12]. Dados clínicos como a doença de base, o motivo e o tempo de internação, foram coletados do prontuário eletrônico.

A avaliação antropométrica foi realizada com o peso e estatura obtidos do prontuário no mesmo dia da entrevista. Após, foram estabelecidos os índices peso para idade (P/I), estatura para idade (E/I) e índice de massa corporal para idade (IMC/I), em escore Z, classificados pelas curvas de crescimento da Organização Mundial da Saúde (OMS) de 2006/2007 [13], através dos softwares WHO Anthro versão 3.2.2 [14] e WHO Anthro Plus [15]. $\mathrm{O}$ diagnóstico nutricional antropométrico foi determinado pelo escore Z do índice IMC/I conforme os pontos de corte propostos pela OMS em 2009. Os pacientes com paralisia cerebral ou síndrome de Down foram avaliados de acordo com curvas de crescimento específicas, conforme proposto por Brooks et al. [16] e Cronk et al. [17], respectivamente. 
As ferramentas ANSG e STRONGkids foram aplicadas durante a entrevista. A classificação final da ANSG foi definida pelas duas pesquisadoras principais através da análise subjetiva de cada item respondido. Já na STRONGkids, o risco nutricional foi definido pela soma da pontuação das quatro questões abordadas no questionário, sendo que o escore igual a zero definiu um baixo risco nutricional, o escore entre 1 e 3 definiu um risco nutricional moderado, e o escore entre 4 e 5 definiu um risco nutricional alto [10].

Para a realização das análises estatísticas, os pacientes foram estratificados de acordo com a doença de base/complicação em 14 diferentes categorias, a saber: cardiologia, nefrologia, cirurgia, pneumologia, neurologia, endocrinologia, gastroenterologia/hepatologia, dermatologia, hematologia, otorrinolaringologia, infectologia, reumatologia, oncologia (incluindo todos os tipos de tumores) e uma categoria com diagnóstico inconclusivo e/ou com outras doenças que não se enquadrassem nas demais. Além disso, as doenças de base/complicações foram divididas em crônicas (diagnósticos de doenças crônicas que não apresentavam risco de vida em curto prazo, ou exacerbação de sintomas normalmente menos intensos nessas condições), agudas (doenças de surgimento repentino, com sintomatologia evidente e tempo de duração definido) e ambas concomitantemente (pacientes com doença crônica cujo motivo de internação constituiu-se de alguma complicação aguda, não necessariamente relacionada à anterior). As variáveis quantitativas foram descritas por média e desvio padrão ou mediana e amplitude interquartílica. As variáveis categóricas foram descritas por frequências absolutas e relativas. $\mathrm{Na}$ comparação de proporções, o teste qui-quadrado de Pearson foi aplicado. Em caso de significância estatística, foi utilizado o teste dos resíduos ajustados. A associação entre o tempo de internação e as classificações da ANSG, STRONGkids e índice IMC/I foi avaliada pelo coeficiente de correlação de Spearman. A concordância dos protocolos ANSG e STRONGkids com o índice IMC/I foi avaliada através do coeficiente Kappa. O nível de significância adotado foi de $5 \%(p<0,05)$. As análises foram realizadas através do programa IBM SPSS Statistics versão 21.0.

\section{RESULTADOS}

Um total de 317 pacientes foram avaliados, sendo que destes, dois apresentavam síndrome de Down e quatro apresentavam paralisia cerebral. Dez pacientes que atendiam aos critérios de inclusão não participaram da pesquisa uma vez que seus responsáveis não concordaram com sua participação. A Tabela 1 apresenta a caracterização da amostra. Os principais motivos de internação foram representados pelas categorias cirurgia $(21,5 \%)$, pneumologia $(20,2 \%)$ e neurologia $(14,5 \%)$.

Tabela 1. Caracterização da amostra que incluiu crianças internadas em um hospital pediátrico de Porto Alegre, Rio Grande do Sul, entre junho e outubro de 2014.

\begin{tabular}{|c|c|}
\hline Variáveis & $\mathrm{n}=317$ \\
\hline Idade (meses) - média $\pm \mathrm{DP}$ & $76,1 \pm 17,5$ \\
\hline \multicolumn{2}{|l|}{ Sexo - n (\%) } \\
\hline Masculino & $179(56,5)$ \\
\hline Feminino & $138(43,5)$ \\
\hline \multicolumn{2}{|l|}{ Cor/Raça - n (\%) } \\
\hline Negra & $23(7,3)$ \\
\hline Branca & $220(69,4)$ \\
\hline Parda & $65(20,5)$ \\
\hline Indígena & $9(2,8)$ \\
\hline \multicolumn{2}{|l|}{ Escolaridade do responsável - $\mathrm{n}$ (\%) } \\
\hline Até $1^{\circ}$ grau completo & $124(39,1)$ \\
\hline Até $2^{\mathrm{o}}$ grau completo & $123(38,8)$ \\
\hline Até superior completo ou mais & $70(22,1)$ \\
\hline \multicolumn{2}{|l|}{ Classe socioeconômica - n (\%) } \\
\hline A, B1, B2 & $199(62,7)$ \\
\hline $\mathrm{C} 1, \mathrm{C} 2, \mathrm{D}, \mathrm{E}$ & $118(37,3)$ \\
\hline $\begin{array}{l}\text { Tempo de internação hospitalar (dias) } \\
\text { - mediana (P25-P75) }\end{array}$ & $6(4-10)$ \\
\hline
\end{tabular}

Segundo a avaliação antropométrica, a maioria dos pacientes $(74,1 \%)$ estavam eutróficos, sendo que $5 \%$ eram desnutridos e 20,8\% apresentavam excesso de peso. Conforme a classificação da ANSG, 84,2\% eram bem nutridos e $15,7 \%$ eram moderadamente e gravemente desnutridos. Já a triagem STRONGkids classificou $24,6 \%$ dos pacientes com baixo risco nutricional e 75,4\% com risco médio e alto (Tabela 2). Em relação aos critérios socioeconômicos, quanto maior a escolaridade dos responsáveis $(\mathrm{p}=0,003)$ e mais alta a classe econômica da família $(\mathrm{p}=0,013)$, maior a probabilidade das crianças estarem em risco nutricional baixo. Não foram encontradas associações estatisticamente significativas dos aspectos socioeconômicos com a avaliação antropométrica e a ANSG.

Encontrou-se associação estatisticamente significativa entre a desnutrição classificada pelo índice IMC/I e o grupo de pacientes com desnutrição moderada e grave pela ANSG $(p<0,001)$. Já a associação da 
Tabela 2. Avaliação nutricional pela avaliação antropométrica, pela Avaliação Nutricional Subjetiva Global e pela Triagem de Risco para Estado Nutricional e Crescimento (STRONGkids). Crianças internadas em um hospital pediátrico de Porto Alegre, Rio Grande do Sul, entre junho e outubro de 2014.

\begin{tabular}{|c|c|}
\hline Variáveis & $\mathrm{n}=317$ \\
\hline Peso atual $(\mathrm{kg})$ - média $\pm \mathrm{DP}$ & $24,2 \pm 7,7$ \\
\hline Estatura $(\mathrm{cm})-$ média $\pm \mathrm{DP}$ & $117,9 \pm 11,6$ \\
\hline IMC/I (escore Z) - média \pm DP & $0,75 \pm 1,72$ \\
\hline \multicolumn{2}{|l|}{ Classificação do índice IMC/I - n(\%) } \\
\hline Desnutrição (escore $Z<-2$ ) & $16(5,0)$ \\
\hline Eutrofia $(-2<$ escore $Z<2)$ & $235(74,1)$ \\
\hline Excesso de peso (escore $Z>$ 2) & $66(20,8)$ \\
\hline P/I (escore Z) - média $\pm D P$ & $0,57 \pm 1,52$ \\
\hline E/I (escore Z) - média $\pm D P$ & $0,06 \pm 1,41$ \\
\hline \multicolumn{2}{|l|}{ Classificação do índice E/I - n(\%) } \\
\hline Baixa estatura (escore $\mathbf{Z}<-2$ ) & $23(7,3)$ \\
\hline Estatura adequada $(-2<$ escore $Z<2)$ & $270(85,2)$ \\
\hline Estatura elevada (escore $Z>2$ ) & $24(7,6)$ \\
\hline \multicolumn{2}{|l|}{ Protocolos de avaliação nutricional - $n(\%)$} \\
\hline \multicolumn{2}{|l|}{ ANSG - n(\%) } \\
\hline Normal/Bem nutrido & $267(84,2)$ \\
\hline Desnutrição moderada & $48(15,1)$ \\
\hline Desnutrição grave & $2(0,6)$ \\
\hline \multicolumn{2}{|l|}{ STRONGkids - $\mathrm{n}(\%)$} \\
\hline Risco nutricional baixo & $78(24,6)$ \\
\hline Risco nutricional médio & $214(67,5)$ \\
\hline Risco nutricional alto & $25(7,9)$ \\
\hline
\end{tabular}

DP, desvio padrão; ANSG, Avaliação Nutricional Subjetiva Global; E/I: estatura para idade; IMC/I: índice de massa corporal para idade; P/I: peso para idade; STRONGkids, Triagem de Risco para Estado Nutricional e Crescimento. avaliação antropométrica com a STRONGkids demonstrou significância estatística entre excesso de peso e risco nutricional baixo, eutrofia e risco nutricional médio, e desnutrição e risco nutricional alto $(\mathrm{p}<0,001)$ (Tabela 3).

Ao correlacionar os resultados das ferramentas ANSG e STRONGkids, assim como o índice IMC/I, com o tempo de internação hospitalar, encontrou-se significância estatística para todos os parâmetros. Quanto mais desnutrido era o paciente segundo a ANSG $\left(r_{s}=0,255 ; p<0,001\right)$ e segundo a avaliação antropométrica $\left(r_{s}=-0,122 ; p=0,031\right)$, e quanto maior era o risco nutricional pela STRONGkids $\left(r_{s}=0,268\right.$; $\mathrm{p}<0,001$ ), maior era o tempo de internação hospitalar. No entanto, as correlações foram de intensidade fraca.

Houve associação significativa entre a doença de base/complicação e a classificação da ANSG ( $p=0,007)$, sendo que os pacientes com somente doença crônica apresentaram maior percentual de eutrofia e aqueles com doença crônica e aguda concomitantemente apresentaram maior percentual de desnutrição grave (Figura 1). Quando buscada associação com os grupos de doenças, 95,7\% dos pacientes com doenças neurológicas estavam eutróficos, e 25\% dos pacientes cardiopatas estavam gravemente desnutridos $\left(\chi^{2}=58,0\right.$; $\mathrm{p}<0,001)$.

Também foi encontrada associação da doença de base/complicação com a classificação da STRONGkids $(\mathrm{p}=0,001)$, sendo que os pacientes com doença aguda e crônica concomitantemente apresentaram risco nutricional alto (Figura 2). Quando avaliada a associação com os grupos de doenças, 43,5\% dos pacientes com doenças neurológicas e 63,6\% dos

Tabela 3. Associação entre o índice de massa corporal para idade e os protocolos de avaliação e triagem nutricional, em 317 crianças internadas em um hospital pediátrico de Porto Alegre, Rio Grande do Sul, entre junho e outubro de 2014.

\begin{tabular}{|c|c|c|c|c|}
\hline \multirow[b]{2}{*}{ Protocolos de avaliação nutricional } & \multicolumn{3}{|c|}{ Classificação do índice IMC/I } & \multirow[b]{2}{*}{ p } \\
\hline & $\begin{array}{c}\text { Desnutrição } \\
\text { n (\%) }\end{array}$ & $\begin{array}{c}\text { Eutrofia } \\
\text { n (\%) }\end{array}$ & $\begin{array}{c}\text { Excesso de peso } \\
n(\%)\end{array}$ & \\
\hline ANSG & & & & $<0,001$ \\
\hline Normal/Bem nutrido & $3(18,8)$ & $200(85,1)$ & $64(97,0)^{*}$ & \\
\hline Desnutrição moderada & $11(68,8)^{*}$ & $35(14,9)$ & $2(3,0)$ & \\
\hline Desnutrição grave & $2(12,5)^{*}$ & $0(0,0)$ & $0(0,0)$ & \\
\hline STRONGkids & & & & $<0,001$ \\
\hline Risco nutricional baixo & $0(0,0)$ & $51(21,7)$ & $27(40,9)^{*}$ & \\
\hline Risco nutricional médio & $12(75,0)$ & $166(70,6)^{*}$ & $36(54,5)$ & \\
\hline Risco nutricional alto & $4(25,0)^{*}$ & $18(7,7)$ & $3(4,5)$ & \\
\hline
\end{tabular}

Comparação entre grupos realizada por meio de teste qui-quadrado de Pearson.

* Associação estatisticamente significativa pelo teste dos resíduos ajustados a 5\% de significância.

ANSG, Avaliação Nutricional Subjetiva Global; IMC/I, índice de massa corporal para idade. 
pacientes do grupo da dermatologia apresentaram risco nutricional baixo; $81,3 \%$ dos pneumopatas e $100 \%$ dos pacientes do grupo da otorrinolaringologia estavam em risco nutricional médio. Além disso, 28,1\% dos pacientes oncológicos e $22,2 \%$ dos pacientes com complicações do grupo da gastroenterologia/ hepatologia estavam em risco nutricional alto $\left(\chi^{2}=73,2\right.$; $\mathrm{p}<0,001)$. A associação das doenças/complicações clínicas com a avaliação antropométrica não apresentou significância estatística $(\mathrm{p}>0,40)$.

Ao avaliar a concordância da avaliação antropométrica com a STRONGkids, encontrou-se resultado estatisticamente significativo, porém de intensidade muito fraca (kappa $=0,148 ; p=0,001)$. Já a concordância com a ANSG foi ainda mais fraca, e sem significância estatística (kappa $=0,043 ; \mathrm{p}=0,063)$.

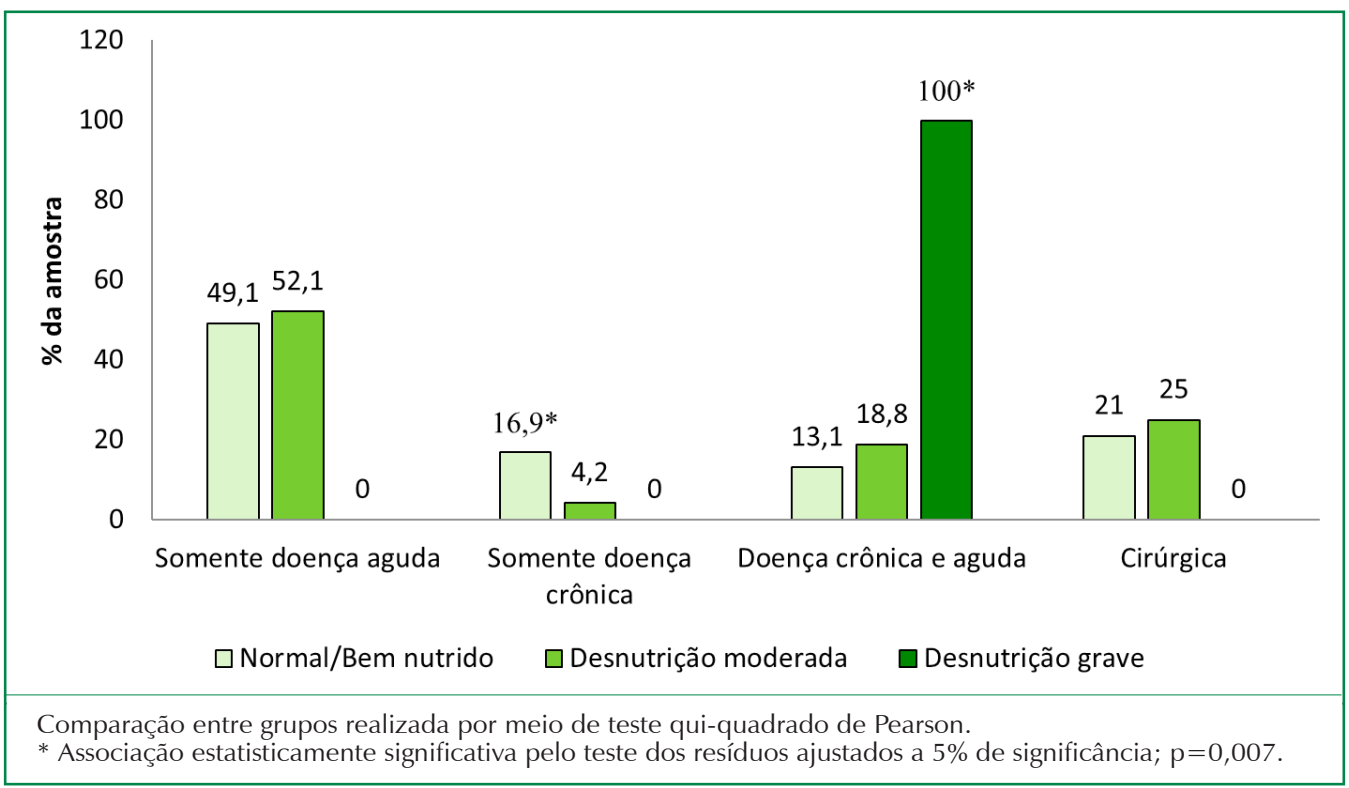

Figura 1. Associação entre a doença de base/complicação e a classificação da Avaliação Nutricional Subjetiva Global, em 317 crianças internadas em um hospital pediátrico de Porto Alegre, Rio Grande do Sul, entre junho e outubro de 2014.

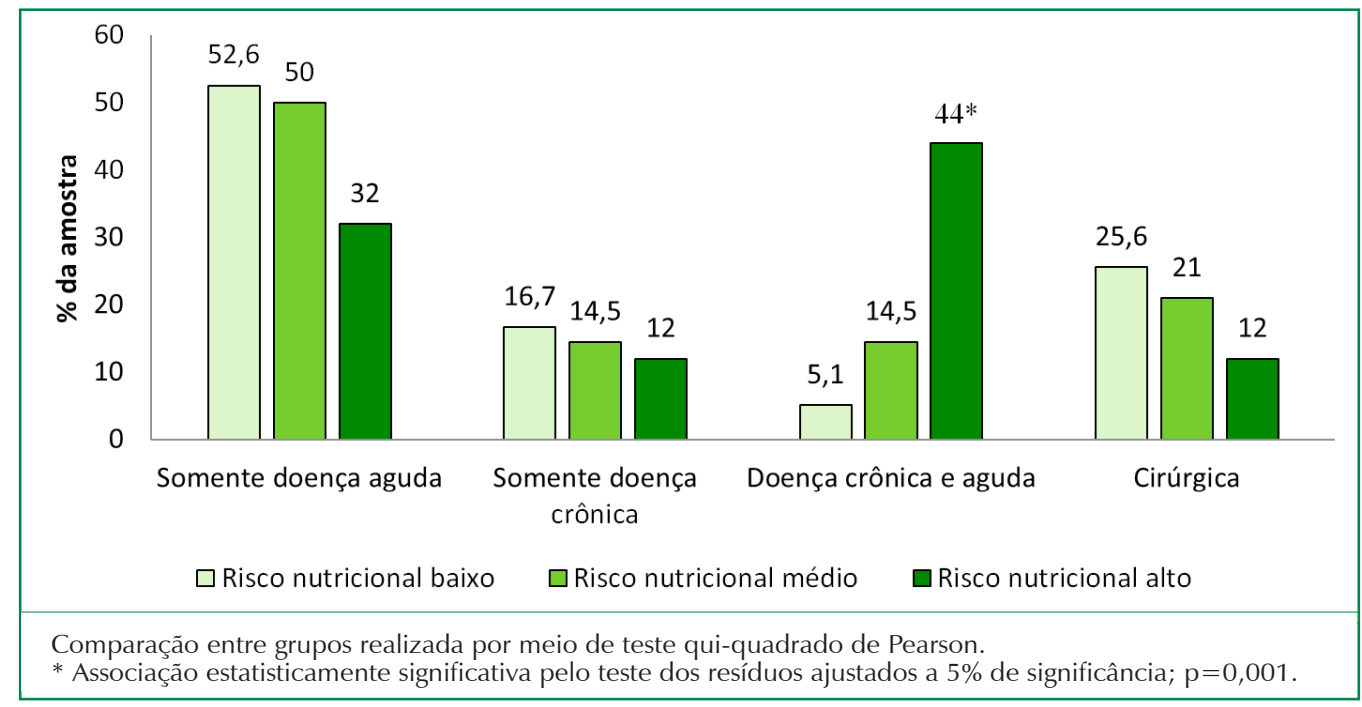

Figura 2. Associação entre a doença de base/complicação e a classificação da Triagem de Risco para Estado Nutricional e Crescimento (STRONGkids). 


\section{DISCUSSÃO}

No presente estudo, todas as ferramentas utilizadas produziram avaliações nutricionais que se relacionaram significativamente (embora apresentando fraca intensidade) com o tempo de internação hospitalar. Os achados de Hulst et al. [10] concordam com estes dados, uma vez que os autores demonstraram que as crianças com baixo risco nutricional ficavam significativamente menos tempo internadas quando comparadas àquelas com risco nutricional moderado ou alto [10]. O estudo de Cao et al. [18] também demonstrou associação entre o alto risco nutricional e o maior tempo de internação hospitalar $(\mathrm{p}<0,001)$ [18]. Outro estudo, que determinou a validade de três ferramentas de triagem nutricional em relação à ANSG (utilizada como padrão ouro) em um hospital pediátrico na Indonésia, também corrobora com os presentes achados ao demonstrar associação entre o alto risco nutricional e o tempo de internação hospitalar $(p=0,004)$ [19]. Já em relação à ANSG, os resultados do presente estudo são semelhantes a um estudo no qual o tempo de internação hospitalar pós-operatório foi maior que o dobro para as crianças gravemente desnutridas (19,0 $\pm 58,8$ dias) em comparação com as eutróficas $(5,3 \pm 5,4$ dias $)$ e as moderadamente desnutridas $(8,4 \pm 11,1 \mathrm{~d})(\mathrm{p}=0,002)$ [8]. Já o estudo de Vermilyea et al. [20] encontrou que o tempo de internação hospitalar não se diferenciou significativamente entre as categorias da ANSG. No entanto, ao combinar os pacientes com desnutrição moderada e grave, o tempo de internação hospitalar no grupo de desnutridos foi seis dias maior, embora ainda sem significância estatística, quando comparado ao grupo de pacientes eutróficos [20].

Foi encontrada uma concordância muito fraca, mas estatisticamente significativa, entre a STRONGkids e o índice IMC/I no presente estudo. Mărginean et al. [21] também encontraram concordância, porém de boa intensidade, entre o risco de desnutrição pela STRONGkids e a desnutrição de acordo com os critérios antropométricos da OMS (kappa $=0,61 ; p=0,001$ ) [21]. Por outro lado, os resultados encontrados por Wiskin et al. [22] não concordam com os presentes achados. Naquele estudo, que comparou quatro ferramentas de triagem nutricional (STRONGkids, Triagem para a Avaliação da Desnutrição Pediátrica, Escore de Yorkhill de Desnutrição Pediátrica e Escore de Risco Nutricional Pediátrico) com os critérios antropométricos para desnutrição propostos pela OMS em 46 crianças com doença inflamatória intestinal, não houve concordância entre as diferentes categorias de risco baseadas nas quatro ferramentas e o grau de desnutrição definido pela avaliação antropométrica [22].

A associação entre a desnutrição classificada pela avaliação antropométrica e o grupo de pacientes com desnutrição moderada e grave pela ANSG também foi encontrada no estudo de Secker e Jeejeebhoy [8], o qual adaptou e testou a validade e a reprodutibilidade da avaliação subjetiva global na população pediátrica. Esse estudo avaliou 175 pacientes cirúrgicos de um mês de vida até 17,9 anos de idade em um hospital do Canadá, tendo mostrado que os pacientes classificados como gravemente desnutridos pela avaliação subjetiva apresentavam menor escore $\mathrm{Z}$ médio para o índice IMC/I, enquanto que aqueles classificados como bem nutridos na ANSG apresentavam um maior escore $\mathrm{Z}$ médio [8]. O estudo que validou a ANSG para uso em Unidades de Terapia Intensiva, realizado com 150 crianças de 31 dias de vida a cinco anos de idade, também confirmou os presentes achados. Esse estudo demonstrou correlação estatisticamente significativa, de intensidade moderada/forte, entre as classificações da ANSG e o índice antropométrico $\mathrm{P} / \mathrm{E}\left(\mathrm{r}_{\mathrm{s}}=-0,57\right.$; $\mathrm{p}<0,0001)$ [20].

Também houve associação estatisticamente significativa entre a triagem nutricional STRONGkids e a avaliação antropométrica no presente estudo. Esses achados concordam com aqueles apresentados por Hulst et al. [10], os quais mostraram que as crianças em risco nutricional alto tinham significativamente menores escores $\mathrm{Z}$ para o índice peso para estatura (P/E) na admissão hospitalar [10]. Da mesma forma, no estudo de Cao et al. [18], o qual avaliou o risco nutricional de 1325 crianças de 29 dias até 17 anos de idade hospitalizadas na China, as médias dos escore $\mathrm{Z}$ para os índices antropométricos P/I, E/I, P/E e IMC/I foram significativamente menores em crianças com alto risco nutricional quando comparadas àquelas com risco nutricional baixo ou moderado [18]. Em outro estudo, que investigou a eficácia da STRONGkids em crianças internadas em 12 hospitais italianos, os pacientes com risco nutricional alto tiveram escores $\mathrm{Z}$ para os índices IMC/I $(-0,79 \pm 2,09 ; \mathrm{p}=0,002)$ e $\mathrm{E} / \mathrm{I}(-1,07 \pm 2,08 ; \mathrm{p}=0,008)$ significativamente menores quando comparados com os outros grupos de risco nutricional [23]. O fato de que, no presente estudo, os pacientes eutróficos pela avaliação antropométrica apresentaram risco nutricional moderado pela STRONGkids pode ser explicado pela baixa prevalência de desnutrição encontrada na amostra. Além disso, essa ferramenta mostrou-se bastante sensível, uma vez que uma única pontuação foi suficiente para definir um risco moderado. 
A prevalência de desnutrição encontrada na amostra estudada foi de $15,7 \%$ de acordo com a ANSG, sendo $15,1 \%$ de desnutrição moderada e $0,6 \%$ de desnutrição grave. Esses valores foram mais baixos quando comparados ao estudo de Secker e Jeejeebhoy [8], que encontraram $36 \%$ e $15 \%$ de desnutrição moderada e grave, respectivamente. Essa diferença de valores pode ser explicada parcialmente pelo tipo de amostra adotada: uma vez que naquele estudo foram analisados apenas pacientes cirúrgicos, presume-se que essas crianças apresentem maiores complicações e por isso estejam em maior risco de desnutrição [8]. Já o presente estudo avaliou pacientes com diagnósticos diversos, tanto clínicos quanto cirúrgicos. Além disso, a diferença de faixa etária avaliada pelos estudos pode ser uma possível explicação para a discrepância nas prevalências encontradas.

No presente estudo, os pacientes que tinham alguma doença de base, além da complicação aguda determinante da internação, apresentaram desnutrição grave pela ANSG e risco nutricional alto pela STRONGkids. Joosten et al. [24] avaliaram o estado nutricional de 424 crianças internadas em 44 hospitais pediátricos na Holanda, tendo encontrado que os pacientes com alguma doença de base apresentaram uma prevalência significativamente maior de desnutrição global e de desnutrição crônica (definida pelo escore $\mathrm{Z}$ do índice $\mathrm{E} / \mathrm{I}<-2$ ) em comparação com aqueles sem doença de base, $28 \%$ vs. $15 \%$ e $18 \%$ vs. $5 \%$, respectivamente $(p=0,004$ e $p<0,001)$ [24]. Esses achados foram semelhantes aos nossos.

Quando analisada a associação da avaliação nutricional subjetiva com os grupos de doenças, foi encontrada maior prevalência de eutrofia no grupo de pacientes com doenças neurológicas, e uma proporção significativa de desnutrição grave no grupo de pacientes com cardiopatia. No que diz respeito ao risco nutricional, os pacientes com doenças neurológicas e dermatológicas apresentaram maior prevalência de baixo risco, sendo que os pacientes dos grupos da oncologia e gastroenterologia/hepatologia apresentaram proporção significativa de risco nutricional alto.

Os achados deste estudo discordam dos resultados encontrados por Joosten et al. [24], cujo estudo encontrou a maior prevalência de desnutrição crônica (31\%) em crianças com distúrbios neurológicos, sendo que aqueles com distúrbios gastrointestinais apresentaram a maior prevalência de desnutrição aguda (18\%) [24]. A maior prevalência de eutrofia no grupo de pacientes com doenças neurológicas, encontrada no presente estudo, pode ser explicada pela grande variabilidade de condições incluídas nessa categoria, abrangendo desde aquelas que implicam maior risco nutricional até as que apresentam um menor risco. Alguns exemplos encontrados nesse grupo de doenças foram epilepsia, trauma cranioencefálico, paralisia cerebral e demais alterações neurológicas. Já o estudo de Cao et al. [18] encontrou associação do alto risco nutricional definido pela STRONGkids com as doenças oncológicas, o que corrobora com os achados do presente estudo, além de ter encontrado associação com as doenças cardíacas e respiratórias [18].

Os pontos fortes deste estudo foram: 1) avaliar crianças na faixa etária de quatro a 8,9 anos de idade, mantendo uma homogeneidade em relação ao estágio de maturação sexual dos participantes [25], o que ajudou a diminuir possíveis vieses quanto à avaliação nutricional dos pacientes; 2) as ferramentas de avaliação nutricional foram discutidas individualmente pelas duas pesquisadoras principais, o que auxiliou na redução da variabilidade dos resultados obtidos. Uma limitação deste estudo é o fato de que nenhuma das ferramentas foi validada para uso no Brasil, tendo sido utilizadas as suas versões traduzidas. Outro ponto importante a ser destacado é que a ANSG classifica os pacientes apenas como bem nutridos, moderadamente ou gravemente desnutridos, o que acaba superestimando a prevalência de eutróficos na amostra e, consequentemente, mascara a prevalência de sobrepeso e obesidade. Já a STRONGkids considera que todos os pacientes possuem risco nutricional, uma vez que, segundo as instruções dessa ferramenta, um escore nulo já define um risco nutricional baixo, não havendo a classificação de pacientes sem risco nutricional. Outra limitação foi a utilização de dados antropométricos obtidos dos prontuários dos pacientes bem como a dificuldade dos informantes em relatar o peso usual da criança de forma precisa, gerando um possível viés de informação.

Concluindo, os resultados de triagem/avaliação nutricional por todos os métodos utilizados no presente estudo associaram-se (embora em fraca intensidade) com o tempo de internação hospitalar. Além disso, a STRONGkids apresentou maior concordância, porém ainda fraca, com a avaliação antropométrica em comparação à ANSG, na admissão hospitalar. No entanto, são necessários mais estudos que avaliem a concordância de ambas as ferramentas com outros métodos objetivos de avaliação nutricional, a fim de que se possa obter o instrumento mais adequado possível para avaliar o risco de deterioração da condição nutricional em crianças hospitalizadas. Também é importante a validação de um questionário de risco nutricional pediátrico para uso no Brasil, para que se possa obter uma avaliação do risco de desnutrição mais adequada às características da população pediátrica brasileira. 


\section{REFERÊNCIAS}

1. Lochs H, Allison SP, Meier R, Pirlich M, Kondrup J, Schneider S, van den Berghe G, Pichard C. Introductory to the ESPEN Guidelines on enteral nutrition: terminology, definitions and general topics. Clin Nutr. 2006;25:180-6. http://dx.doi.org/10.1016/j.clnu.2006.02.007

2. Joosten KFM, Hulst JM. Malnutrition in pediatric hospital patients: Current issues. Nutrition. 2011;27:133-7. http://dx.doi.org/10.1016/j. nut.2010.06.001

3. Bejon P, Mohammed S, Mwangi I, Atkinson SH, Osier F, Peshu N, Newton CR, Maitland K, Berkley JA. Fraction of all hospital admissions and deaths attributable to malnutrition among children in rural Kenya. Am J Clin Nutr. 2008;88:1626-31. http://dx.doi.org/10.3945/ ajcn.2008.26510

4. Hartman C, Raanan S, Hecht C, Koletzko B. Malnutrition screening tools for hospitalized children. Curr Opin Clin Nutr Metab Care. 2012;15:303-9. http://dx.doi.org/10.1097/MCO.0b013e328352ded4

5. Dal Bosco S. Terapia nutricional pediátrica. 1aㅡ ed. São Paulo: Atheneu, 2010.

6. Mahdavi AM, Safaiyan A, Ostadrahimi A. Subjective vs. objective nutritional assessment study in children: a cross-sectional study in the northwest of Iran. Nutr Res. 2009;29:269-74. http://dx.doi.org/10.1016/j.nutres.2009.03.009

7. Sermet-Gaudelus I, Poisson-Salomon AS, Colomb V, Brusset MC, Mosser F, Berrier F, Ricour C. Simple pediatric nutritional risk score to identify children at risk of malnutrition. Am J Clin Nutr. 2000;72:64-70.

8. Secker DJ, Jeejeebhoy KN. Subjective global nutritional assessment for children. Am J Clin Nutr. 2007;85:1083-9.

9. McCarthy H, McNulty H, Dixon M, Eaton-Evans MJ. Screening for nutrition risk in children: the validation of a new tool. J Hum Nutr Diet. 2008;21:395-6. http://dx.doi.org/10.1111/j.1365-277X.2008.00881_31.x

10. Hulst JM, Zwart H, Hop WC, Joosten KFM. Dutch national survey to test the STRONGkids nutritional risk screening tool in hospitalized children. Clinical Nutrition. 2010;29:106-11. http://dx.doi.org/10.1016/j.clnu.2009.07.006

11. Gerasimidis K, Keane O, Macleod I, Flynn DM, Wright CM. A four-stage evaluation of the Paediatric Yorkhill Malnutrition Score in a tertiary paediatric hospital and a district general hospital. Br J Nutr. 2010;104:751-6. http://dx.doi.org/10.1017/S0007114510001121

12. ABEP - Associação Brasileira de Empresas de Pesquisa [Internet]. Critério de Classificação Econômica Brasil. 2012 [cited 2014 out 27 ]. Available from: http://www.abep.org/codigosCondutas.aspx

13. WHO Multicentre Growth Reference Study Group. WHO child growth standards based on length/height, weight and age. Acta Paediatr Suppl. 2006;450:76-85.

14. Software for assessing growth and development of the world's children. WHO Anthro for personal computers [programa de computador] Versão 2011. Geneva: World Health Organization; 2010. [cited 2014 out 27]. Available from: http://www.who.int/childgrowth/software/ en/

15. Software for assessing growth of the world's children and adolescents. WHO AnthroPlus for personal computers [programa de computador]. Versão 2009. Geneva: World Health Organization, 2009. [cited 2014 out 27]. Available from: http://www.who.int/growthref/tools/en/

16. Brooks J, Day S, Shavelle R, Strauss D. Low weight, morbidity, and mortality in children with cerebral palsy: new clinical growth charts. Pediatrics. 2011;128:e299-e307. http://dx.doi.org/10.1542/peds.2010-2801

17. Cronk C, Crocker AC, Pueschel SM, Shea AM, Zackai E, Pickens G, Reed RB. Growth charts for children with Down syndrome: 1 month to 18 years of age. Pediatrics. 1988;81:102-10.

18. Cao J, Peng L, Li R, Chen Y, Li X, Mo B, Li X. Nutritional risk screening and its clinical significance in hospitalized children. Clinical Nutrition. 2014;33:432-36. http://dx.doi.org/10.1016/j.clnu.2013.06.009

19. Wonoputri N, Djais JTB, Rosalina I. Validity of nutritional screening tools for hospitalized children. J Nutr Metab. 2014;2014:143649. http://dx.doi.org/10.1155/2014/143649

20. Vermilyea S, Slicker J, El-Chammas K, Sultan M, Dasgupta M, Hoffmann RG, Wakeham M, Goday PS. Subjective global nutritional assessment in critically ill children. JPEN J Parenter Enteral Nutr. 2013;37:659-66. http://dx.doi.org/10.1177/0148607112452000

21. Mărginean O, Pitea AM, Voidăzan S, Mărginean C. Prevalence and assessment of malnutrition risk among hospitalized children in Romania. J Health Popul Nutr. 2014;32:97-102.

22. Wiskin AE, Owens DR, Cornelius VR, Wootton SA, Beattie RM. Paediatric nutrition risk scores in clinical practice: children with inflammatory bowel disease. J Hum Nutr Diet. 2012;25:319-22. http://dx.doi.org/10.1111/j.1365-277X.2012.01254.x

23. Spagnuolo MI, Liguoro I, Chiatto F, Mambretti D, Guarino A. Application of a score system to evaluate the risk of malnutrition in a multiple hospital setting. Ital J Pediatr. 2013;39:81. http://dx.doi.org/10.1186/1824-7288-39-81

24. Joosten KF, Zwart H, Hop WC, Hulst JM. National malnutrition screening days in hospitalized children in The Netherlands. Arch Dis Child. 2010;95:141-45. http://dx.doi.org/10.1136/adc.2008.157255

25. Tanner JM. Growth at adolescence: with a general consideration of the effects of hereditary and environmental factors upon growth and maturation from birth to maturity. 2nd edition. Oxford: Blackwell Scientific Publications; 1962. 